\begin{tabular}{|l|l|l||}
\hline \multicolumn{2}{|c|}{ PublisherInfo } \\
\hline \hline PublisherName & $:$ & BioMed Central \\
\hline \hline PublisherLocation & $:$ & London \\
\hline \hline PublisherImprintName & $:$ & BioMed Central \\
\hline \hline
\end{tabular}

\title{
CT imaging, lower inflection points and PEEP
}

\begin{tabular}{|l|l|l||}
\hline \multicolumn{2}{|c||}{ ArticleInfo } \\
\hline \hline ArticleID & $:$ & 4072 \\
\hline \hline ArticleDOI & $:$ & $10.1186 /$ ccf-1999-1021 \\
\hline \hline ArticleCitationID & $:$ & 1021 \\
\hline \hline ArticleSequenceNumber & $:$ & 9 \\
\hline \hline ArticleCategory & $:$ & Paper Report \\
\hline \hline ArticleFirstPage & $:$ & 1 \\
\hline \hline ArticleLastPage & $:$ & 4 \\
\hline \hline & & RegistrationDate : 1999-7-22 \\
\hline ArticleHistory & $:$ & OnlineDate \\
\hline \hline ArticleCopyright & $:$ & Current Science Ltd1999-7-22 \\
\hline \hline ArticleGrants & $:$ & \\
\hline \hline ArticleContext & $:$ & 130541111 \\
\hline \hline
\end{tabular}




\section{Keywords}

acute lung injury, pressure volume curve, lower inflection point, lung morphology

\section{Comments}

This is a complex and detailed study, which appears to have been meticulously performed. Though limited by small numbers of patients, it adds considerably to the observation that there are two distinct patterns of ALI which this study suggests can be differentiated by lung compliance, PVC and CT scan. Notably, the two patterns observed in this study were unrelated to the etiology, which is at odds with other published work suggesting that this difference is entirely attributable to the nature of ALI, ie whether it is direct or indirect. Perhaps the most interesting observation is the effect of PEEP on the relative proportions of overdistented and non-aerated lung, which suggest that a lower PEEP (approx 10 $\mathrm{cmH}_{2} \mathrm{O}$ ) is the best compromise between recruitment and barotrauma, in the more compliant and regionally affected patients without a LIP on their PVCs, but higher PEEP (approx $15 \mathrm{cmH}_{2} \mathrm{O}$ ) is of benefit in the less compliant and diffusely affected patients with a LIP on their PVCs. The clinical relevance of this study is brought home by the seemingly dramatic but statistically not significant difference in mortality between the groups, with a LIP 5/8 (63\%) versus $1 / 6(17 \%)$ without a LIP. To take this further, this study needs either to be repeated in another center, or continued to include more patients.

\section{Introduction}

In patients with acute lung injury (ALI) there is frequently a characteristic change in the pressure volume curve (PVC). It has been argued that a PVC should be performed in all such patients to guide therapy, such that positive end expiratory pressure (PEEP) is set above the lower inflection point (LIP) of the curve, and plateau pressure set below the upper inflection point. The theory is that PEEP at this level will recruit and retain alveolar units, and that plateau pressure at such a level will minimize barotrauma. However, it is often found that patients with ALI have no LIP on their PVC. To date, the morphological explanation for this finding has not been investigated, nor the effects of PEEP in ALI patients with or without an LIP on their PVC. Given the advances in computerized tomography (CT) it is now possible to image these patients to investigate these theories. 


\section{Aims}

To establish the lung morphology that correlates with the presence or absence of a LIP on the PVC of patients with ALI and to investigate the effects of PEEP on such patients.

\section{Methods}

A total of 14 patients with ALI were studied prospectively. All patients were treated identically, having the same invasive monitoring, ventilation and drug therapy. Respiratory and cardiovascular parameters, PVCs and thoracic CT were all performed at zero end expiratory pressure (ZEEP) and at two levels of PEEP. The first level, PEEP1, was set at the LIP $+2 \mathrm{cmH}_{2} \mathrm{O}$, or in the absence of a LIP at $10 \mathrm{cmH}_{2} \mathrm{O}$. The second, PEEP2, was set at $\mathrm{LIP}+7 \mathrm{cmH}_{2} \mathrm{O}$, or $15 \mathrm{cmH}_{2} \mathrm{O}$, respectively. All measurements were taken once the patient had reached a steady state.

PVCs were obtained by performing 'study breath' occlusions, during which the airway and esophageal pressures generated by pre-set tidal volumes were measured, as previously described by Levy and coworkers (J Crit Care 1989, 4:83-89). Esophageal pressures were recorded via a transduced water filled catheter. PVCs were then constructed for the total respiratory system, the lungs and the chest wall.

Following these measurements, patients underwent thoracic CT within $6 \mathrm{~h}$. Transportation to the scanner was controlled and a period of $15 \mathrm{~min}$ stability ensured, following arrival, prior to scanning. The scanning protocol was identical for all patients, with cessation of mechanical ventilation barring ZEEP/ PEEP during image acquisition. A qualitative assessment of the lung morphology was made by a single, 'blinded' radiologist. Total lung volumes were measured and the percentage of overdistended, normally aerated, poorly aerated and non-aerated lung were determined by previously validated, automated methods, which depend upon CT density determination. Gas-tissue ratio was also calculated, again the method used has been previously validated.

\section{Results}

The 14 patients were a heterogenous sample of ALI cases, whose clinical characteristics suggest they are a typical group. Eight of the 14 patients had a LIP on their total respiratory system and lung PVC. Of these, only two also exhibited an LIP on their chest wall PVC. Patients with a LIP tended to be younger, had a higher mortality rate and lung injury severity score. There was a marked difference between the lung morphology on CT between the two groups, which was statistically significant. Patients with a LIP had diffuse hyperdensities, whereas patients without a LIP had hyperdensities localized predominantly in the lower lobes. Under ZEEP conditions, patients with a LIP had significantly lower lung compliance. Mean LIP was $9 \mathrm{cmH}_{2} \mathrm{O}$. PEEP induced alveolar recruitment in all patients although the pattern differed 
between the two groups. Near maximal recruitment was achieved at PEEP1 in patients without a LIP whereas significant recruitment continued at PEEP2 in patients with a LIP. PEEP induced lung overdistension was only observed in patients without a LIP. PEEP did not significantly change the volume of poorly aerated lung in any of the patients.

\section{Discussion}

Quantitative analysis of thoracic CT in this sample of patients found a similar amount of alveolar damage in all cases but two distinct patterns of distribution. These findings were matched by characteristic PVCs and response to PEEP. The combination of quantifyinglung volumes and composition (densitometry) by CT offers useful clinical information with important therapeutic consequences. The underlying reasons for the development of each of these two patterns is unclear, but does not appear to be related to underlying etiology.

It has been argued that areas of non-aerated lung on CT scan have two distinct etiologies, small airway collapse and true alveolar collapse. It has been demonstrated that re-opening collapsed airways requires far less pressure than re-opening alveolar units. This model explains the biphasic volume recruitment caused by PEEP. This study implies that the presence of a LIP appears to correlate with a greater degree of small airway collapse. The induction of overdistention in patients without a LIP is explained by the significantly greater degree of normally aerated lung in these patients.

\section{References}

1. Vieira SRR, Puybasset L, Lu Q, et al: A scanographic assessment of pulmonary morphology in acute lung injury. Significance of the lower inflection point detected on the lung pressure-volume curve. Am J Respir Crit Care Med. 1999, 159: 1612-1623. 